\title{
Der Deutsche Qualifikationsrahmen für lebenslanges Lernen
}

\author{
Ziel ist die Anerkennung von Kompetenzen, egal ob sie durch \\ formelles oder informelles Lernen erworben wurden
}

Eberhard Funk

Dr. Eberhard Funk ist Historiker, Betriebswirt und Politikwissenschaftler. Er arbeitet als wissenschaftlicher Referent für Planung, Steuerung und Qualifizierung der sozialen Arbeit und der sozialen Dienste im Deutschen Verein für öffentliche und private Fürsorge mit dem Arbeitsschwerpunkt »Qualifizierung und Soziale Berufe«.

E-Mail funk@deutscher-verein.de
Der vor fünf Jahren begonne Prozess für einen "Deutschen Qualifikationsrabmen " tritt bald in seine entscheidende Phase. Die Soziale Arbeit muss sich in diese Diskussion einschalten, wenn sie nicht den Anschluss an internationale Entwicklungen verlieren will.

Weltweit arbeiten rund einhundert Staaten an Qualifikationsrahmen der (beruflichen) Bildung oder haben sie schon erarbeitet, um ihre Systeme transparenter und letztlich leistungsfähiger zu machen.

Die Europäische Union ist prominent an diesem Prozess beteiligt. Im April 2008 verabschiedeten Europäisches Parlament und Europäischer Rat einen »Europäischen Qualifikationsrahmen für lebenslanges Lernen « (EQR). Er verfolgt u. a. das Ziel, die Mobilität in der Ausund Weiterbildung in Europa zu befördern. Transparenz und Qualität der Bildungssysteme und Bildungsangebote sollen europaweit gewährleistet werden. Er orientiert sich an Kompetenzen und beruflicher Handlungsfähigkeit. Seine acht Referenzniveaus decken die gesamte Bandbreite von Qualifikationen ab, die in Form von Lernergebnissen beschrieben werden: Letztere zeigen an, was ein Lernender nach Abschluss eines Lernprozesses weiß, versteht und in der Lage ist zu tun.

Der »Europäische Qualifikationsrahmen für lebenslanges Lernen « beruht auf drei Säulen: Kenntnissen, Fähigkeiten und Kompetenzen. Er versteht sich als Brücke zwischen formalem, nonformalem und informellem Lernen und bezieht damit durch Erfahrungen gewonnene - Lernergebnisse mit ein; jedes Qualifikationsniveau soll grundsätzlich auf verschiedenen Bildungs- und Karrierewegen erreichbar sein.

In diesem Kontext hat in Deutschland seit Juni 2007 der Arbeitskreis Deutscher Qualifikationsrahmen (AK DQR), dem rund 30 Mitglieder aus den Bereichen Wirtschaft, Wissenschaft, Gewerkschaften, Freie Wohlfahrtspflege, Vertretungen von Bundesländern sowie des Bundesinstituts für Berufsbildung angehören, unter dem Ko-Vorsitz des Bundesministeriums für Bildung und Forschung und der Kultusministerkonferenz einen Deutschen Qualifikationsrahmen erarbeitet. Im Februar 2009 hat der Arbeitskreis einen »Diskussionsvorschlag eines Deutschen Qualifikationsrahmen für lebenslanges Lernen « vorgelegt. Seine Elemente wurden bis Herbst 2010 validiert. Auf der 17. Sitzung des Arbeitskreises am 10. November 2010 wurde er im Konsens beschlossen. Er besteht aus einem Einführungstext, einer Matrix und einem Glossar. Vereinbart, aber noch nicht umgesetzt, ist die Einbeziehung nonformal und informell erworbener Kompetenzen in die Niveaustufen des Deutschen Qualifikationsrahmen.

Der bildungsbereichsübergreifende Deutsche Qualifikationsrahmen kann ein wichtiges Instrument für eine höhere Durchlässigkeit des Bildungssystems und der Gleichwertigkeit zwischen beruflicher, allgemeiner und hochschulischer Bildung werden. Grundsätzlich sollen alle Kompetenzniveaus des Deutschen Qualifikationsrahmens auf schulischen, betrieblichen, hochschulischen und beruflichen Bildungs- und Karrierewegen erreichbar sein und prinzipiell keine Niveaus für bestimmte Qualifikationen reserviert werden.

Der Deutsche Qualifikationsrahmen soll helfen, Inhalte der Ausbildungen lernergebnis- (outcome-) orientiert zu formulieren und (Aus-) Bildungen (inhaltlich und zeitlich) flexibler zu gestalten. Überall soll die Anschlussfähigkeit sichergestellt werden, wofür zertifizierbare Instrumente für die Erfassung und Bewertung von Kompetenzen entwickelt und Kompetenzstandards der entsprechenden 
Stufe des Deutschen Qualifikationsrahmens zugeordnet werden müssen.

Der Deutsche Qualifikationsrahmen stellt ein Referenzsystem dar, an dem sich bestehende Bildungsergebnisse orientieren; es besteht, wie der Europäische Qualifikationsrahmen für lebenslanges Lernen, aus acht Referenzniveaus, die für Bildungsbehörden und Bildungseinrichtungen, aber auch den Einzelnen als gemeinsamer Bezugspunkt fungieren. Die acht Niveaus decken sämtliche Qualifikationen $a b$, von Basisqualifikationen bis zur akademischen und beruflichen Ausund Weiterbildung. An die Stelle bildungsbiografisch geprägter Abschlüsse treten Kompetenzen. Kompetenzen werden hierbei hierarchisch, nicht praxisorientiert definiert.

Während einige europäische Länder (z. B. Schweiz, Frankreich, Finnland und Dänemark) bei der Erarbeitung ihrer nationalen Qualifikationsrahmen von einem umfassenden Bildungsverständnis ausgingen, in dem formale, nonformale und informelle Bildungsprozesse gleichermaßen Eingang fanden, ging Deutschland stufenweise vor: In der Validierungsphase wurden zunächst anerkannte Berufe (formale Qualifikationsprofile) als Maßstab für die Zuordnung zu den Stufen des Deutscher Qualifikationsrahmen gewählt. Leitidee war hierbei »employability« (Beschäftigungs- und Berufsfähig- keit); eine Kompetenzbasierung wurde dadurch erst einmal verschoben; sie würde damit erst bei einer Neuordnung von Berufen angewandt werden. In die Validierungsphase haben somit entgegen der angestrebten Lernweg- und Lernort-Unabhängigkeit von Kompetenzbestimmung auch prozessorientierte Kompetenzen und Input-Kriterien Eingang gefunden. Fragen der Mobilität und formaler Lernprozesse standen im Vordergrund.

\section{Viersäulig statt dreisäulig}

Der Deutsche Qualifikationsrahmen hat sich für ein viersäuliges System entschieden, beruhend auf Fachkompetenz, die aus Wissen und Fertigkeiten besteht, und personaler Kompetenz, die sich aus Sozialkompetenz und Selbstständigkeit zusammensetzt.

Mit der Bestimmung und starken Gewichtung von Handlungskompetenz setzt sich der Deutsche Qualifikationsrahmen vom dreisäuligen System des Europäischen Qualifikationsrahmens für lebenslanges Lernen ab. Hierbei wendet er andere Kriterien an als der Europäische Qualifikationsrahmen für lebenslanges Lernen - er beschreibt allgemein-persönliche Kompetenzen, basierend auf beruflicher Handlungsfähigkeit, anstelle von Kompetenzen in der beruflichen Bildung -,

\section{Was ist eigentlich der Deutsche Qualifikationsrahmen?}

Im Oktober 2006 haben sich das Bundesministerium für Bildung und Forschung und die Kultusministerkonferenz darauf verständigt, gemeinsam einen Deutschen Qualifikationsrahmen für lebenslanges Lernen zu entwickeln und die relevanten Akteure in diesen Prozess einzubinden. Ebenso soll eine breite Fachöffentlichkeit in die Diskussion einbezogen werden.

Ausgangspunkt für diese Entscheidung ist die Empfehlung des Europäischen Parlaments und des Rats zur Einrichtung des Europäischen Qualifikationsrahmens, der am 23. April 2008 in Kraft trat. Dieser soll bis 2010 in allen Ländern der Europäischen Union mit Hilfe nationaler Regelungen umgesetzt werden. Er bildet als Referenzrahmen für lebenslanges Lernen die Leistungen der jeweiligen nationalen Bildungssysteme auf europäischer Ebene in acht Niveaustufen ab. Damit dient er dazu, Lernergebnisse aus allen Bildungsbereichen international verständlicher und vergleichbarer zu machen. In dieser Funktion als Übersetzungsinstrument zwischen den Bildungs- und Qualifikationsssystemen der Mitgliedstaaten trägt er dazu bei, dass Arbeitnehmer und Lernende ihre Qualifikationen auch über die eigenen Ländergrenzen hinweg nutzen können.

Damit die Ergebnisse des deutschen Bildungssystems EU-weit Anerkennung finden, müssen sie dem Europäischen Qualifikationsrahmen angemessen zugeordnet werden können. Dazu ist es notwendig, die nationalen Bildungsleistungen durch einen Deutschen Qualifikationsrahmen zu definieren.

Internet http://www.deutscherqualifikationsrahmen.de

woraus sich eine grundsätzliche Gleichwertigkeit von fachlicher und personaler Kompetenz ergibt.

Nicht in die Matrix des Deutschen Qualifikationsrahmens aufgenommen wurden Handlungskompetenzen, die vorhanden, aber im konkreten Arbeitsablauf nicht nachgefragt werden (Ausdauer, Verlässlichkeit, Aufmerksamkeit, Genauigkeit, intellektuelle Kompetenz) sowie ethische Dispositionen (normative, ethische und religiöse Reflexivität, interkulturelle und interreligiöse Kompetenz, gelebte Toleranz, demokratische Verhaltensweise), die lediglich Eingang in den Einführungstext gefunden haben, jedoch durchlaufende Dimensionen aller acht Niveaus sind.

Die Validierungsphase hat in vier exemplarisch ausgewählten Berufs- und Tätigkeitsfeldern (den Metall- und Elektroberufen, dem Handel, dem Gesundheitswesen und dem IT-Bereich) Kriterien für die berufsbegleitende zertifizierte Qualifikation und Verfahren und Standards für Äquivalenzfeststellungen festgelegt. Insbesondere wurden die Niveaustufen identifiziert, auf denen die exemplarisch betrachteten Qualifikationen jeweils auf der Basis des definierten Kompetenzbegriffs anzusiedeln sind, das »Feintuning «.

Obwohl großer wissenschaftlicher Sachverstand vertreten war, handelt es sich letztlich doch um einen politischen Prozess, in dem Setzungen auch Verhandlungssache sind. Generell lässt sich sagen, dass hierbei die berufliche Bildung und insbesondere das Handwerk erfolgreicher agiert haben, als der akademische Bereich. Der Deutscher Qualifikationsrahmen gliedert sich in folgende Stufen:

Stufe 1: Basisqualifikationen

Stufe 2: qualifizierter Hauptschulabschluss, Ausbildungsreife

Stufe 3: zweijährige Berufsausbildung, Fachabschluss, Mittlerer Abschluss

Stufe 4: dreijährige Berufsausbildung, qualifizierter Fachabschluss, Fachhochschulreife

Stufe 5: dreijährige Berufsausbildung mit Funktionserfahrung, fachgebundene und allgemeine Hochschulreife (1)

Stufe 6: Meister, Techniker, Bachelor

Stufe 7: MBA, Master

Stufe 8: Promotion 


\section{Nonformal und informell erworbener Qualifikationen}

Für die Anerkennung nonformal und informell erworbener Kompetenzen wurden in die Diskussion zum Deutschen Qualifikationsrahmen zwei Gutachten (2) einbezogen. Sie legen ein (fünfschrittiges) Verfahren nahe, das aus Information und Beratung, Ermittlung, Bewertung, Validierung sowie Zertifizierung bestehen könnte. In diesen Zusammenhang passt auch der in den Freiwilligendiensten entwickelte »ProfilPASS « (wie auch EUROPASS), der in Form eines Portfolios Belege über individuelle Fähigkeiten und Kompetenzen zusammenstellt.

Weitere konkrete Verfahren für die $\mathrm{Zu}$ lassung zu Bildungsgängen oder Prüfungen liegen vor, an denen man anknüpfen könnte, beispielsweise in Form von bereits gesetzlich geregelten NichtschülerPrüfungen für allgemeinbildende $\mathrm{Ab}$ schlüsse (nach $\mathbb{\$} 45$, Absatz 2 Berufsbildungsgesetz oder $\mathbb{S} 37$, Absatz 2 Handwerksordnung), unterschiedlichen Formen von Externenprüfungen, Studiermöglichkeiten ohne Abitur, Begabtenprüfungen, Einstufungsprüfungen, Probestudien im Hochschulbereich und anderen Anrechnungsverfahren.

Alle entsprechenden Verfahren werden bislang allerdings nur punktuell eingesetzt und sind kaum durchschaubar. Hier könnte die Matrix des Deutschen Qualifikationsrahmens zu größerer Transparenz beitragen und bestehende (in formalen Verfahren erworbene) Zertifikate (wie beispielsweise den Europäischen Computerführerschein, Sprachenzertifikate, Expert-Zertifikate der Volkshochschulen, Schweißer-, Gabelstapler- oder Gefahrgutfahrer-Zertifikate oder die Hersteller-Zertifikate im IT-Bereich) einbeziehen.

\section{Zunehmende Bedeutung lebenslangen Lernens}

Die Förderung des lebenslangen Lernens wird auch in Deutschland als eine zentrale Antwort auf bildungspolitische Fragen der Gegenwart gesehen. Es soll eine eigenverantwortliche Lebensgestaltung und gesellschaftliche Teilhabe ebenso ermöglichen, wie die Stärkung des Wirtschaftsraums und die Realisierung von Chancengleichheit. Zudem soll gene- rell das Bildungsniveau erhöht werden, wobei neue Zugänge zu Bildung und Lernen geöffnet, Barrieren zwischen Bildungsbereichen abgebaut und verborgene Potenziale identifiziert und nutzbar gemacht werden sollen. Durch Anknüpfung an bisheriges Lernen soll »Doppellernen « vermieden und Lernzeiten verkürzt werden, so dass die Durchlässigkeit zwischen Bildungsgängen erhöht und Abschlüsse anschlussfähig werden.

Sowohl der "Bologna-Prozess « wie der durch den Vertrag von Maastricht in der beruflichen Bildung angestoßene »Ko- gen von Ländern mit differenziertem und transparentem Hochschulzugang für Berufserfahrene nahelegen.

\section{European Credit System for Vocational Education and Training}

European Credit System for Vocational Education and Training (ECVET) ist ein mit dem Europäischen Qualifikationsrahmen für lebenslanges Lernen korrespondierendes europäisches Leistungspunkte-

\section{»Die Wohlfahrtsverbände müssen nun die Qualifikationen ibrer Berufe kompetenzorientiert beschreiben, um die sozialen und die Gesundheits- berufe adäquat in den Deutschen Qualifikationsrahmen einordnen zu können «}

penhagen-Prozess « bieten unter dem Stichwort "Soziale Dienste«, Verfahren und Modelle der Anerkennung von Lernleistungen an, die über die Wertschätzung von bisher Erlerntem zum Weiterlernen und zum Beschreiten neuer Lern- und Berufswege anregen.

Lebenslanges Lernen kann zur besseren Integration von formal Geringqualifizierten in den Arbeitsmarkt beitragen, indem Benachteiligte ihre zumeist informell erworbenen Kompetenzen, anerkennen lassen können; sie kann zur Nutzung der Potenziale von Migrantinnen und Migranten mittels erleichterter Anerkennung von im Ausland erworbenen beruflichen Qualifikationen für (adäquate) Beschäftigung und dauerhafte Integration in den Arbeitsmarkt beitragen und die berufliche Mobilität erhöhen. Sie unterstützt Querund Seiteneinstiege in Berufslaufbahnen und trägt damit zur Stärkung individueller beruflicher Entwicklungswege und einer anforderungsgerechten Besetzung von Stellen und erhöht voraussichtlich die Studierberechtigtenquote, wie Erfahrun- system in der beruflichen Bildung, das in Gremien der Europäischen Union erarbeitet wurde. Es soll Transparenz, Vergleichbarkeit, Transferierbarkeit und wechselseitige Anerkennung von beruflichen Qualifikationen und Kompetenzen auf verschiedenen Niveaus fördern.

Qualifikationen werden dabei nicht durch den für ihren Erwerb notwendigen Aufwand, sondern durch die erzielten Lernergebnisse und Kompetenzen beschrieben. Um eine Kompatibilität mit möglichst vielen auf nationaler Ebene etablierten Bewertungsverfahren zu erreichen, können in die Definition der ECVET-Niveaus unterschiedliche Bewertungskriterien einfließen wie Dauer und Art, Ziele und/oder Ergebnisse der Bildungsmaßnahme, erforderliche Kompetenzen, um bestimmte Tätigkeiten ausüben zu können, die Position einer Qualifikation in der Berufshierarchie und die Einordnung bestehender Niveaus aufgrund von Entsprechungsnachweisen.

Diese Heterogenität der Bewertungskriterien kann allerdings dazu führen, 
dass gleiche Qualifikationen in verschiedenen Ländern unterschiedlichen Niveaus zugeordnet werden. Die Entwicklung von ECVET setzt daher einen europäischen Konsens zu Überprüfungsverfahren erworbener Kompetenzen und zur Qualitätssicherung voraus; es kann die wechselseitige Anerkennung voranbringen und fehlende einheitliche Berufsbezeichnungen in Europa »übersetzen «.

ECVET wurde vom Europäischen Parlament im Dezember 2008 beschlossen; es soll ab 2012 »schrittweise « angewandt werden. Wie das Credit-Punkte-System des Bologna-Prozesses der Hochschulen (ECTS) sieht auch ECVET die Vergabe von 60 Credits jährlich (bei einer Vollzeitausbildung) vor. Generell stellt sich das Problem einer systematischen Verknüpfung von ECTS mit ECVET. Auch wenn die Bundesrepublik sich für die Übernahme von ECVET entschieden hat, bleibt ungeklärt, wie ein tarifierbares System entstehen soll und wer Lerneinheiten und Punkte anerkennen soll. Jedenfalls muss es "konform « mit den bisherigen Berufsbildungssystemen eingeführt werden.

Einzelne Domänen werden eigene Methoden und zertifizierbare Instrumente für die Erfassung und Einordnung von Kompetenzen entwickeln müssen (in Form von Leittexten, Aufgabenbeschreibungen etc.). Das vorgesehene Deutsche Leistungspunktesystem der beruflichen Bildung (DECVET) wird sich hieran orientieren müssen. Wie ECVET soll DECVET beruflich-fachlich outcomeorientiert sein, und unabhängig vom Input, der Dauer der Ausbildung und den Zeugnissen werden.

\section{Verhaltener Widerstand der Hochschulen}

Vorbehalte am Deutschen Qualifikationsrahmen gibt es vonseiten der Hochschulrektorenkonferenz, die - obwohl seit Beginn am Prozess um den Deutschen Qualifikationsrahmen beteiligt - neuerdings kritisiert, dass die derzeitige Fassung des Deutscher Qualifikationsrahmen dem Anspruch nicht gerecht würde, ein bildungsgangübergreifendes Transparenzinstrument zu sein. Ihr ist die spezifische wissenschaftliche Problemlösungskompetenz auf den höheren Niveaus zu undeutlich formuliert und auf den Niveaus
6 bis 8 das Verhältnis von beruflichen und hochschulischen Bildungsgängen zu unklar. Befürchtet werden negative Konsequenzen für den Hochschulbereich, da sich der Stellenwert von Kompetenzen, die in einem Studium erworben werden, im Vergleich zu den in der Berufsbildung erworbenen Kompetenzen verringere.

Befürchtet wird, dass der Druck auf Öffnung des Zugangs zum Hochschulbereich so groß wird, dass Bewerberinnen und Bewerber zugelassen werden müssten, die nicht für ein Studium geeignet sind, wodurch Studierende überfordert würden, was zur Senkung des Niveaus von Lehre und Studium führen könnte. Zudem werden durch den Prozess zum Europäischen Qualifikationsrahmen für lebenslanges Lernen und zum Deutschen Qualifikationsrahmen voraussichtlich auch Studiengänge auf dem Kompetenzniveau 5 (Short Cycle) seitens der Hochschulen oder der Weiterbildung geschaffen werden, die bisher im Deutschen Hochschulwesen unbekannt waren.

\section{Berufsprinzip}

Ebenso strittig ist die Bedeutung von Outcome-Orientierung im Zusammenhang mit dem deutschen Berufsprinzip. In der Debatte zum Deutschen Qualifikationsrahmen haben insbesondere die Sozialpartner, aber auch die Bundesregierung am "Berufsprinzip « festgehalten. (3) So sei zu beachten, dass »die Ausrichtung an Kompetenzen und beruflicher Handlungsfähigkeit (nicht bedeute), dass anstelle gesellschaftlich normierter und standardisierter Lernprozesse (z. B. Ausbildungsordnungen) Kombinationen von beliebigen Lernvorgaben treten « (4). Die berufliche Bildung will so ihre tradierte Struktur (den angeblichen "Königsweg « aus dualer Ausbildung und Aufstiegsfortbildung) erhalten. Lediglich die Übergänge mittels einer Entsäulung des Bildungssystems stehen in ihrem Fokus; einer Entsäulung allerdings im wesentlichen nur zwischen Hochschulbildung und beruflicher Bildung und nicht unbedingt zwischen dem sogenannten »Übergangssystem « und der beruflichen Bildung. So betreibt sie die (Teil-) Anerkennung von beruflich erworbenen Qualifikationen durch die Hochschulen, steht aber, wie die Bundesregierung einer von vielen Staaten angestrebten Akkumulierung von
Qualifikationen zu einem Beruf ablehnend gegenüber.

Bisher hat sich die berufliche Bildung nicht auf plausible Übergangsregelungen zwischen der Berufsausbildungsvorbereitung und der Berufsausbildung im dualen System, sowie einer positiven Bewertung der Qualifikationen von beruflichen Vollzeitschulen im dualen System verständigen können. Eine solche Position drückt sich auch im Widerstand der Sozialpartner gegen eine differenzierte (kompetenzbasierte) Zuordnung und Spreizung der dualen Berufe in den Deutschen Qualifikationsrahmen aus; stattdessen präferieren sie einheitliche Einordnungen (wie beispielsweise auch die Hochschulen in ihrem Bereich). Generell bleibt in der deutschen Debatte das Stichwort "Modularisierung « ein Reizwort.

Daher ist es ein Erfolg des Deutschen Qualifikationsrahmens, mit der Beschreibung von Basiskompetenzen auch Menschen ohne qualifizierten Hauptschulabschluss in den Deutscher Qualifikationsrahmen zu integrieren. Die auf Niveau 1 aufgenommenen »Basiskompetenzen « umfassen hierbei die Bandbreite von Teilqualifikationen auf dem Niveau des Hauptschulabschlusses bis hin zur Erfüllung individuell festgelegter Bildungsziele.

\section{Aufgaben, Folgerungen und nächste Schritte}

Der weitere Zeitplan des Deutschen Qualifikationsrahmens ist eng gestrickt. Die Ergebnisse der Validierungsphase sollen von den Staatssekretären der Bundesländer im ersten Quartal 2011 beraten werden. Danach wird ein Handbuch erarbeitet, das Verfahren zur Kompetenzfeststellung beschreibt; nach diesem werden die vorhandenen Qualifikationen zu den Niveaus 1 bis 8 zugeordnet. Parallel soll eine Strategie der Einbeziehung nonformalen und informellen Lernens erarbeitet werden, um bis zum vierten Quartal 2011 deren Einordnung in den Deutschen Qualifikationsrahmen durch zwei oder drei Arbeitsgruppen zu ermöglichen. Die Bundesarbeitsgemeinschaft der Freien Wohlfahrtsverbände hat ihre Bereitschaft erklärt, hieran mitzuwirken.

Parallel dazu wird eine wissenschaftliche Begleitforschung installiert und ein breites (wissenschaftliches und politisches) Konsultationsverfahren um den 
Deutschen Qualifikationsrahmen organisiert werden, um breite Akzeptanz in der Gesellschaft und eine europäische Kompatibilität zu erreichen. Der Arbeitskreis Deutscher Qualifikationsrahmen und die Bund-Länder-Koordinierungsgruppe Deutscher Qualifikationsrahmen sollen den Prozess weiter begleiten.

Noch im Jahr 2011 sollen die Qualifikationen und Kompetenzen von »zuständigen Stellen « der jeweiligen Stufe des Deutscher Qualifikationsrahmen zugeordnet werden und ab 2012 auf allen neuen Zeugnissen und Qualifikationsnachweisen vermerkt werden und mittels eines Verweises auf das zutreffende Niveau des Europäischen Qualifikationsrahmen für lebenslanges Lernen mit diesem verknüpft werden. Es wird besonders Aufgabe jeder Domäne sein, ihre jeweiligen Kompetenzen zu regeln, einschließlich des erforderlichen Punktesystems und geeignete Verfahren der Plausibilitätsprüfung und der Qualitätssicherung zu entwickeln. (Das vorgesehene Berufsbildungspisa wird sich voraussichtlich u. a. mit Kompetenzfeststellungsverfahren befassen.) Unklar bleibt vorerst, wer die "zuständige Stelle« bei den Sozialund Gesundheitsberufen sein wird.

\section{Konsequenzen für die Soziale Arbeit}

Vor den Wohlfahrtsverbänden steht die Aufgabe, die Qualifikationen ihrer Berufe kompetenzorientiert $\mathrm{zu}$ beschreiben und zu definieren, welche Kompetenzen für welche Tätigkeit Voraussetzung sein müssen, um die sozialen und Gesundheitsberufe adäquat in den Deutschen Qualifikationsrahmen einordnen zu können. Zudem muss festgelegt werden, wer in diesem Zusammenhang die »zuständige Stelle « ist, und wie und wer gegebenenfalls fehlende Kompetenzen und Mo-

\section{Die Ausbildung in sozialen Berufen}

ist in Deutschland inhaltlich wie formal vielgestaltig und führt zu Abschlüssen unterschiedlicher Ausrichtung und Wertigkeit je nach Bildungsgangund Bundesland. Sie vermittelt Kenntnisse und Kompetenzen, die in den Tätigkeitsfeldern der sozialen Berufe bzw. den entsprechenden Tätigkeiten benötigt werden. Da die sozialen Berufe, sofern die Heilberufe nach Art. 74 Abs. 1 Grundgesetz (GG) nicht mitgemeint sind, landesrechtlichen Regelungen unterliegen, ist die Ausbildung in Deutschland nicht einheitlich. Das System der landesrechtlich geregelten Ausbildung ist allerdings durch länderübergreifende Rahmenvereinbarungen der Kultusministerkonferenz (KMK), wie z. B. zur Ausbildung und Prüfung von Erzieher/innen oder zu den Fachschulen, koordiniert.

Die Ausbildungsstrukturen in den sozialen Berufen sind fast so vielgestaltig wie das System der sozialen Berufe selbst. Die Spanne der Qualifikationsniveaus reicht von kurzen Lehrgängen (z. B. European Care Licence) über ein-, zwei- und dreijährige Berufsausbildungen bis hin zu Masterstudiengängen (Bachelor-Master-Stystem) an Fachhochschulen und Universitäten (Hochschulen) und anderen Spezialisierungen. Die Abschlussbezeichnungen sind nicht alle einheitlich und dieselbe Berufsbezeichnung lässt nicht unbedingt darauf schließen, dass es sich um den gleichen Beruf mit gleicher Ausbildung handelt. In den aktuellen Berufsstatistiken des Statistischen Bundesamtes und der Bundesanstalt für Arbeit beruhen die Bezeichnungen der sozialen Berufe nur auf den Tätigkeitsfeldern, nicht auf den Ausbildungsniveaus. Unter den Bezeichnungen Sozialarbeiter/in oder Heilpädagog/in finden sich beispielsweise Berufstätige mit Hochschul-, Fachschul- oder sonstigem beruflichen Abschluss. Zur Vielfalt der Ausbildung trägt auch bei, dass die DDR andere Ausbildungen und Abschlussbezeichnungen führte als die Bundesrepublik. Aktuell kommt hinzu, dass sich, angestoßen durch allgemeine Umwälzungen im Bildungswesen auf europäischer und nationaler Ebene (Bolognaprozess, Kopenhagenprozess, Qualifikationsrahmen), viele Ausbildungen wandeln und neue Ausbildungsgänge entstehen.

Die seit den 1970er-Jahren bestehenden Hochschulstudiengänge mit Diplomabschluss in der Sozialarbeit/Sozialpädagogik, Pädagogik und teils auch Heilpädagogik sind schon weitgehend durch Bachelorstudiengänge ersetzt. In der Ausbil- dung zur Sozialen Arbeit an den Fachhochschulen ist dieser Wandel schon vollständig vollzogen. Neben den Bachelorstudiengängen ist aber auch eine Vielzahl an spezialisierten Masterstudiengängen entstanden, nicht nur im Sozialmanagement und der klinischen Sozialarbeit, sondern auch in der Erziehungswissenschaft, Pflege, Heilpädagogik, in einzelnen Arbeitsfeldern der Sozialen Arbeit wie Gemeinwesenarbeit, Jugendhilfe, in der Beratung und für die Seelsorge. Die meisten dieser Masterstudiengänge sind weiterbildende Masterstudiengänge, d. h. sie setzen eine zwischenzeitliche einschlägige Berufstätigkeit voraus. Teilweise gibt es auch konsekutive Masterstudiengänge, die im direkten Anschluss an den vorlaufenden Bachelorstudiengang studiert werden können. In den letzten Jahren kamen neue Bachelorstudiengänge in den Gesundheitsberufen Physiotherapie, Logopädie und Ergotherapie sowie in der Ausbildung der Erzieher/ innen dazu. Die Abschlussbezeichnungen und Curricula dieser Studiengänge gleichen sich im Großen und Ganzen, weisen teils aber auch deutliche Unterschiede auf. Inzwischen gibt es auch einige duale Pflegestudiengänge, die ein akademisches Studium mit der Berufssausbildung kombinieren.

Die Ausbildungsanforderungen in den etablierten sozialen Berufen wurden in den letzten Jahren angehoben. Hierbei spielen berufsständische Professionalisierungsbestrebungen eine wichtige Rolle, jedoch auch hoheitliche Bestimmungen (z. B. der Beschluss der Jugendministerkonferenz vom 12.5.2005) sowie der allgemein zunehmende Bezug auf wissenschaftliche Methoden und Erkenntnisse (Sozialwissenschaften, Gesundheitswissenschaften, Pflegewissenschaften und bezugswissenschaftliche Erkenntnisse). Die Curricula orientieren sich unter dem Einfluss der europäischen Bildungspolitik zunehmend an Kompetenzen bzw. den Lernergebnissen (Outcomeorientierung). Damit könnte es auch international zu einer etwas größeren Konvergenz der Ausbildungen und Berufsbilder kommen.

Peter Buttner

Quelle: Deutscher Verein für öffentliche und private Fürsorge e. V. (Hg.): Fachlexikon der sozialen Arbeit. 7. Auflage. Nomos Verlagsgesellschaft, Baden-Baden 2011. Ca. 1.200 Seiten. 44,- Euro. ISBN 978-3-8329-5153-5. Seite 70 f. 
dule feststellen oder einfordern kann. Offene Fragen bleiben:

- Gelingt eine plausible Zuordnung allgemeinbildender Abschlüsse? Strittig an dem Vorschlag des Schulausschusses der Kultusministerkonferenz ist vor allem die Zuordnung des Abiturs auf der Niveaustufe 5 und damit die Zulassungsvoraussetzungen für die Hochschule. Nach Ansicht der beruflichen Bildung widerspricht eine solche $\mathrm{Zu}$ ordnung den Niveaubeschreibungen des Deutschen Qualifikationsrahmens und den verabredeten Definitionen ebenso, wie dem Zuordnungsverhalten anderer europäischer Länder. Sie fordert daher, dass - falls das Abitur doch wider alle Plausibilität - der Niveaustufe 5 zugeordnet bleibt, alle drei- und dreieinhalbjährigen Berufsausbildungen ebenfalls diesem Niveau zugeordnet werden müssen.

- Muss für eine Qualifikation »als Ganzes « eine Übereinstimmung vorliegen (»full fit «) oder reicht eine ziemlich genaue (»best fit «) aus? Ungeklärt ist auch, wo doppeltqualifizierende Abschlüsse landen (beispielsweise Berufsqualifizierung plus Fachhochschulreife). Wo wird beispielsweise die Referendarausbildung zugeordnet?

- Umstritten bleibt, ob bei Zuordnungsfragen die EU-Anerkennungsrichtlinie 2005/36/EG über die Anerkennung von Berufsqualifikationen für die geregelten Berufe (insbesondere die Gesundheitsberufe) zwingend angewandt werden muss, die, mittels eines fünfstufigen, abschließenden Systems, andere Anerkennungsverfahren als im Europäischen Qualifikationsrahmen vorsieht. Auf jeden Fall muss die Anerkennungsrichtlinie überarbeitet werden, um sie mit dem Europäischen Qualifikationsrahmen für lebenslanges Lernen kompatibel zu machen.

- Ein Nebenaspekt der Diskussion zum Deutschen Qualifikationsrahmen ist, dass der Deutsche Qualifikationsrahmen auch einen Ausweg aus der in der Bundesrepublik gescholtenen Bildungsberichterstattung der OECD aufzeigen würde, indem nicht mehr die Akademikerquote, sondern die Abschlüsse auf Niveau 5 oder 6 des Europäischen und des Deutschen Qualifikationsrahmens verglichen würden.

- Die Hauptfrage bleibt aber und lässt sich erst in der Perspektive beantwor- ten: Bildet die Einstufung beruflicher Qualifikationen und Kompetenzen im Deutschen Qualifikationsrahmen lediglich ein Transparenz- und Übersetzungssystem oder ein diagnostisches Instrument? Aktuell begründet die Einstufung auf einem bestimmten Niveau keine, wie auch immer definierten Ansprüche (auch wenn andere Staaten hier teilweise anders verfahren). Gleichwohl wird sie möglicherweise Auswirkungen auf Tarif- und Eingruppierungsfragen haben und ein wichtiges Instrument im Diskurs der Bildungssysteme werden.

Die Antwort auf die Frage, ob die Validierungskonzepte von Europäischem und Deutschem Qualifikationsrahmen (nur) einer größeren Transparenz dienen, oder wir den Beginn eines Validierungssystems erleben, das sich parallel zum formalen Bildungssystem entwickelt, lässt sich erst im Verlauf des Prozesses beantworten. Dieses Parallelsystem würde auf denselben Standards und Zertifikaten beruhen wie das bisherige System, würde aber weitere Verfahren und Kompetenznachweise integrieren.

Vielleicht erleben wir auch gerade den Beginn eines neuen kompetenzbasierten Systems, in das alle Kompetenzen und Standards unter Einbeziehung des nichtformalen und informellen Lernens einfließen und welches das bisherige, auf Erfassung formaler Qualifikationen in formalen Verfahren beruhende, System ersetzt.

\section{Anmerkungen}

(1) Die Einstufung des Abiturs auf Niveau 5 durch den Schulausschuss der Kultusministerkonferenz wird von fast allen Beteiligten aus systematischen und Vergleichsgründen abgelehnt. Bleibt sie doch auf Niveau 5, müssen andere Qualifikationen, insbesondere die drei- und dreieinhalbjährigen Qualifikationen auf Niveau 4 ebenfalls auf Niveau 5 eingeordnet werden.

(2) Dehnbarkeit, P./Seidel, S./StammRiemer, K.: Die Einbeziehung von Ergebnissen informellen Lernens in den DQR. Eine Kurzexpertise, Bonn/Hannover 2010, sowie Gutschow, K. u. a.: Anerkennung von nicht formal und informell erworbenen Kompetenzen, Bericht an den Hauptausschuss des BIBB, Bonn 2010.
(3) Wachstum, Bildung, Zusammenhalt, Koalitionsvertrag CDU, CSU, FDP, 17. Legislaturperiode.

(4) Hermann Nehls: in: Berufsbildung in Wissenschaft und Praxis, 2008, S. 50.

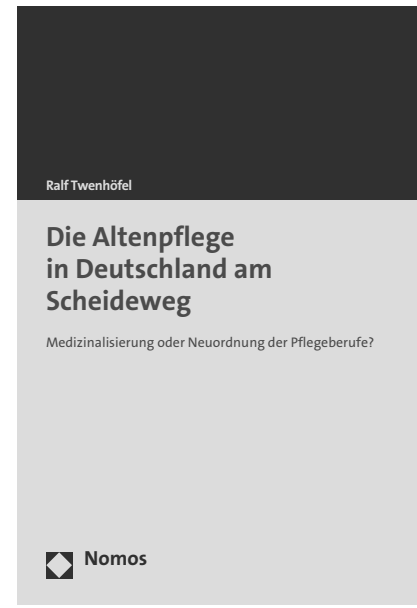

\section{Die Altenpflege in Deutschland am Scheideweg}

Medizinalisierung oder Neuordnung der Pflegeberufe?

Von Prof. Dr. Ralf Twenhöfel 2011, 176 S., brosch., 29,-€, ISBN 978-3-8329-6031-5

Die Untersuchung wendet sich gegen einseitige Bestrebungen einer „Medizinalisierung“ der Altenpflege. Diese würde dadurch auf „Krankenpflege für alte Menschen" reduziert. Systemtheoretisch zeigt der Autor Erfordernisse und Möglichkeiten einer umfassenden Professionalisierung des Berufs, die Aufgaben der "Sorge“ („Caring“) einbezieht, auf.

\section{Nomos}

